\title{
EDITORIAL
}

\section{Do bacteria have a role in asthma development?}

\author{
J. Armann and E. von Mutius
}

\section{$\mathbf{T}$} he relationship between the microbial world and asthma is not well understood. Although we know that the most potent triggers of wheezing attacks are viruses, we do not understand whether and how they contribute to disease onset and progression. Even less is known about the relationship between asthma and bacteria.

The clearest evidence stems from studying asthma exacerbations or wheezing episodes. In clinical studies viruses can be detected in up to $90 \%$ of such episodes [1], with rhinovirus being most commonly identified, followed by respiratory syncytial virus (RSV) in the first years of life. Other viruses such as parainfluenza, metapneumovirus, coronavirus, adenovirus, influenza and enteroviruses have also been implicated, mostly in older patients. In $\sim 10 \%$ of episodes multiple viruses were found [2]. Most recently, the H1N1 virus has been reported to be more prevalent among asthmatics than other patients [3]. In a recent high-risk birth cohort, children with wheezing illnesses triggered by rhinovirus infections early in life were at risk for the subsequent development of asthma up to 6 yrs of age [2].

However, it remains unclear whether viral infections are causal factors for the new onset of disease by impacting on the host's immune response or whether they merely unmask a host's underlying susceptibility to develop asthma by triggering exacerbations. It has been proposed that asthmatics may have subtle deficiencies in their immune response resulting in reduced antiviral activity and increased susceptibility for viral infections, especially of the lower respiratory tract. CORNE et al. [4] recruited 76 cohabiting couples where only one partner had asthma and compared the frequency, severity and duration of rhinovirus infection between them. The authors found that asthmatics develop more severe and longer lasting respiratory symptoms following rhinovirus infections than their spouses [4]. Message et al. [5] experimentally infected adult asthmatics and healthy controls with rhinovirus and assessed respiratory symptoms, lung function, airway inflammation, T-cell phenotypes and cytokine production. In their study asthmatics had a higher viral load, deficient interferon (IFN)- $\gamma$ and interleukin (IL)-10 responses and an augmented IL-4, IL-5, and IL-13 response following infection compared with healthy controls [5]. Others studies have shown deficiencies in type-I IFN production among asthmatics [6].

Allergy/Pulmonology, University Children's Hospital, Munich, Germany.

CORRESPONDENCE: J. Armann, Allergy/Pulmonology, Ludwig-Maximilians-Universität München, Lindwurmstraße 4, D-80336 Munich, Germany. E-mail: jakob.armann@med.Imu.de
Much less is known about the role of bacteria in the pathogenesis of asthma. Serological evidence of an immune response to Streptococcus pneumoniae, Haemophilus influenzae and Moraxella catarralis can be found in $\sim 20 \%$ of wheezing children [7]. Mycoplasma pneumoniae and Chlamydia pneumoniae were identified in 5-25\% of children with asthma exacerbations [8]. A significantly greater reduction in asthma symptoms and a larger improvement from baseline lung function was found in adult patients treated with a macrolide for acute asthma exacerbations [9]. However, there are conflicting data from other studies. Therefore, GRAHAM et al. [10] concluded that there was insufficient evidence to favour treatment of acute asthma episodes with antibiotics. In addition, for the therapy of chronic asthma, treatment with macrolides has not consistently been shown to significantly improve outcomes [11].

Even less is known about the impact of airway colonising bacteria on asthma development. The fact that a viral infection is most often detected when there is serological evidence of bacterial infection [7] suggests that bacteria may boost immunoglobulin (Ig) levels secondary to viral infections when access to the underlying immune system is gained via the inflamed mucosa. However, many interactions between microbes and humans do not result in infections and there is increasing evidence that exposure to commensal bacteria is essential for the development and activity of the host's immune system [12]. With the emergence of culture independent molecular methods for the detection of bacteria the human bacterial microbiota has been shown to be more diverse than expected. It does not only involve the gut and skin [13], but also the lower respiratory tract [14] which was previously thought to be sterile. HiLTY et al. [14] performed bronchoscopy on 24 adults (chronic obstructive pulmonary disease $n=5$, asthma $n=11$, healthy controls $\mathrm{n}=8$ ) and on 20 children (asthma $\mathrm{n}=13$, controls $\mathrm{n}=7$ ). Every $\mathrm{cm}^{2}$ contained a mean of 2,000 bacterial genomes. Interestingly, Haemophilus spp. were much more frequent in asthmatics and chronic obstructive pulmonary disease patients, whereas controls were more likely to be colonised with Prevotella spp. Whether this particular pattern of colonisation is attributable to treatment with inhaled steroids or is a distinct feature of asthma remains to be elucidated.

In a Danish birth cohort, colonisation with $H$. influenzae early in life was a risk factor for subsequent asthma. BISGAARD et al. [15] obtained aspirates from the hypopharyngeal region of healthy infants at the age of 1 month, cultured the aspirates for $H$. influenzae, S. pneumoniae, M. catarralis and Staphylococcus aureus and prospectively followed the children until 5 yrs of age. Colonisation of the upper airways with $S$. pneumonia, $H$. influenzae or $M$. catarrhalis, but not S. aureus, was a predictor of recurrent wheeze and asthma at 5 yrs of age [15]. 
In this issue of the European Respiratory Journal, Hollams et al. [16] report an association between asthma and levels of specific IgE antibodies against $S$. aureus enterotoxin (SAE) and surface antigens of $H$. influenzae and S. pneumonia. It has previously been shown that IgE antibodies are not only directed towards inhalant and food allergens, but also towards microbes. WELLIVER et al. [17] reported IgE responses to RSV in children with bronchiolitis. Furthermore, these authors showed that this IgE response to RSV early in life was related to recurring wheezing, but not to lung function and allergic sensitisation at age $7-8$ yrs [18]. Thus, these findings may suggest that $\operatorname{IgE}$ antibodies to RSV occur independently of atopy and may be indicative of an ongoing asthmatic process. IgE antibodies towards bacterial antigens have also been described previously. Specific IgE to $H$. influenzae and S. pneumonia have been found in the serum of approximately a third of atopic children and asthmatic adults, but unlike in the study by Hollams et al. [16] they were all related to a subject's atopic status [19].

In the study by Hollams et al. [16] a positive association between IgE levels against SAE and an inverse relationship between IgE levels to $H$. influenzae and $S$. pneumonia surface antigens and asthma in a cohort of 1,380 teenagers is reported. At first glance, these findings may suggest that a subject's $\operatorname{IgE}$ immune response may be indicative of harmful and protective bacterial exposures for the development of asthma. However, SAE and $H$. influenzae and $S$. pneumonia surface antigens interact quite differently with the immune system. SAE acts as superantigen which can directly activate T-cells and can shift the cytokine pattern towards a T-helper (Th) 2 profile. Thus, IgE antibodies to SAE may be regarded as seconding the atopic process as suggested by the authors. In turn, $H$. influenzae and S. pneumonia surface antigens have to be processed and presented by antigen presenting cells (APC) to activate T-cells and, thus, may not directly induce a Th2 response.

However, the effects of Th2 cytokines, such as IL-4 and IL-13, seem far more complex than just impacting on $\operatorname{IgE}$ production [20]. As Hollams et al. [16] discuss there is some evidence that Th2 cytokines may suppress other pro-inflammatory cytokines, such as tumour necrosis factor- $\alpha$ and IL-1, as well as pro-inflammatory mediators such as prostaglandin, thereby counter regulating increased Th1 immune responses [21]. Thus, bacterial IgE to $H$. influenzae and S. pneumonia surface antigens might merely reflect such a downregulating Th2 response and may be regarded as a marker for an overall balanced immune response towards airway colonising bacteria. Such balanced immunity could prevent an excessive response to the transmucosal leakage and uptake of bacterial antigens by APCs in the context of exacerbations induced by viral infections. In this scenario a Th2 response would merely be indicative of an augmented Th1 response brought about by certain bacterial exposures in the airways. Hence, production of IgE antibodies towards H. influenzae and S. pneumonia would be associated with less asthma and, thus, indirectly corroborate the notion of an increased asthma risk by airway colonisation with "asthmatogenic" bacterial species.

However, the role and regulation of $\operatorname{IgE}$ antibodies towards microbial antigens compared to allergens is far from understood. The levels of IgE antibodies to $H$. influenzae and $S$. pneumonia were very low in this study. Furthermore, the findings do not agree with the previous report discussed previously showing a positive relationship between IgE to $H$. influenzae and S. pneumonia with atopy $[16,19]$. Future studies will have to elucidate a potential dualistic role of Th2 responses as proposed by the authors. This notion implies that strong exposures to asthmatogenic bacteria induce a harmful Th1 response which must be counterbalanced by Th2 immunity. Although the concept of asthmatogenic airway colonisation is very attractive, the supporting data are still limited [14, 15]. The future will certainly bring about more studies in this evolving field and will elucidate the complex relationship between asthma and the bacterial world.

\section{STATEMENT OF INTEREST}

A statement of interest for E. von Mutius can be found at www.erj. ersjournals.com $/ \mathrm{misc} /$ statements.dtl

\section{REFERENCES}

1 Papadopoulos NG, Xepapadaki P, Mallia P, et al. Mechanisms of virus-induced asthma exacerbations: state-of-the-art. A GA ${ }^{2} \mathrm{LEN}$ and InterAirways document. Allergy 2007; 62: 457-470.

2 Jackson DJ, Gangnon RE, Evans MD, et al. Wheezing rhinovirus illnesses in early life predict asthma development in high-risk children. Am J Respir Crit Care Med 2008; 178: 667-672.

3 Jain S, Kamimoto L, Bramley AM, et al. Hospitalized patients with 2009 H1N1 influenza in the United States, April-June 2009. N Engl J Med 2009; 361: 1935-1944.

4 Corne JM, Marshall C, Smith S, et al. Frequency, severity, and duration of rhinovirus infections in asthmatic and non-asthmatic individuals: a longitudinal cohort study. Lancet 2002; 359: 831-834.

5 Message SD, Laza-Stanca V, Mallia P, et al. Rhinovirus-induced lower respiratory illness is increased in asthma and related to virus load and Th1/2 cytokine and IL-10 production. Proc Natl Acad Sci USA 2008; 105: 13562-13567.

6 Walton RP, Johnston SL. Role of respiratory viral infections in the development of atopic conditions. Curr Opin Allergy Clin Immunol 2008; 8: 150-153.

7 Lehtinen P, Jartti T, Virkki R, et al. Bacterial coinfections in children with viral wheezing. Eur J Clin Microbiol Infect Dis 2006; 25: 463-469.

8 Korppi M. Management of bacterial infections in children with asthma. Expert Rev Anti Infect Ther 2009; 7: 869-877.

9 Johnston SL, Blasi F, Black PN, et al. The effect of telithromycin in acute exacerbations of asthma. N Engl J Med 2006; 354: 1589-1600.

10 Graham V, Lasserson T, Rowe BH. Antibiotics for acute asthma. Cochrane Database Syst Rev 2001; 3: CD002741.

11 Richeldi L, Ferrara G, Fabbri LM, et al. Macrolides for chronic asthma. Cochrane Database Syst Rev 2005; 4: CD002997.

12 Dethlefsen L, McFall-Ngai M, Relman DA. An ecological and evolutionary perspective on human-microbe mutualism and disease. Nature 2007; 449: 811-818.

13 Ahmed S, Macfarlane GT, Fite A, et al. Mucosa-associated bacterial diversity in relation to human terminal ileum and colonic biopsy samples. Appl Environ Microbiol 2007; 73: 7435-7442.

14 Hilty M, Burke C, Pedro H, et al. Disordered microbial communities in asthmatic airways. PLoS One 2010; 5: e8578.

15 Bisgaard H, Hermansen MN, Buchvald F, et al. Childhood asthma after bacterial colonization of the airway in neonates. $N$ Engl J Med 2007; 357: 1487-1495.

16 Hollams EM, Hales BJ, Bachert C, et al. Th2-associated immunity to bacteria in teenagers and susceptibility to asthma. Eur Respir J 2010; 36: 509-516. 
17 Welliver RC, Kaul TN, Ogra PL. The appearance of cell-bound IgE in respiratory-tract epithelium after respiratory-syncytial-virus infection. N Engl J Med 1980; 303: 1198-1202.

18 Welliver RC, Duffy L. The relationship of RSV-specific immunoglobulin $\mathrm{E}$ antibody responses in infancy, recurrent wheezing, and pulmonary function at age 7-8 years. Pediatr Pulmonol 1993; 15: 19-27.

19 Pauwels R, Verschraegen G, van der Straeten M. IgE antibodies to bacteria in patients with bronchial asthma. Allergy 1980; 35: 665-669.
20 de Waal Malefyt R, Figdor CG, Huijbens R, et al. Effects of IL-13 on phenotype, cytokine production, and cytotoxic function of human monocytes. Comparison with IL-4 and modulation by IFN-gamma or IL-10. J Immunol 1993; 151: 6370-6381.

21 Hart PH, Vitti GF, Burgess DR, et al. Potential antiinflammatory effects of interleukin 4: suppression of human monocyte tumor necrosis factor alpha, interleukin 1, and prostaglandin E2. Proc Natl Acad Sci USA 1989; 86: 3803-3807. 